\title{
Nahrungsergänzungsstoffe bei beginnender Alzheimer-Erkrankung nicht wirksam
}

Fragestellung: Hat eine Behandlung mit Nahrungsergänzungsstoffen einen günstigen Einfluss auf eine beginnende Alzheimer-Erkrankung?

Hintergrund: In den letzten Jahren hat sich immer stärker herauskristallisiert, dass der pathologische Prozess, der am Ende zu einer manifesten Alzheimer-Erkrankung führt, wahrscheinlich bereits zehn bis zwanzig Jahre vor der klinischen Manifestation beginnt. Vor diesem Hintergrund hat ein Paradigmenwechsel stattgefunden, indem jetzt Anstrengungen unternommen werden, die Erkrankung bereits entweder im Prodromalstadium oder bei milder klinischer Ausprägung zu behandeln. Es gibt eine Reihe von epidemiologischen Studien, die nahelegen, dass neben der Behandlung von vaskulären Risikofaktoren eine gesunde Ernährung einen protektiven Effekt bezüglich der Alzheimer-Demenz haben könnte.

In der Vergangenheit waren drei kleinere Studien zum Einsatz von Nahrungsergänzungsstoffen bei beginnender Alzheimer-Erkrankung durchgeführt worden. Davon hatten zwei ein positives und eine ein negatives Ergebnis gezeigt. Diese Untersuchungen erstreckten sich allerdings über lediglich zwölf Monate. Vor diesem Hintergrund sollte in der längeren und größeren LipiDiDiet-Studie untersucht werden, inwieweit dieses Behandlungskonzept bei Patienten im Prodromalstadi-

Soininen $\mathrm{H}$, Solomon A, Visser PJ et al. 24-month intervention with a specific multinutrient in people with prodromal Alzheimer's disease (LipiDiDiet): a randomised, double-blind, controlled trial. Lancet Neurol 2017; 16: $965-75$ um der Alzheimer-Erkrankung einen günstigen Einfluss haben könnte.

Patienten und Methodik: Die LipiDiDiet-Studie war als eine 24-monatige randomisierte kontrollierte, dop- pelblinde Parallelgruppenstudie angelegt, die in Zentren in Schweden, Finnland, Deutschland und in den Niederlanden durchgeführt wurde. Eingeschlossen wurden Patienten mit nachweisbaren Biomarkern im Liquor, Veränderungen in der Kernspintomografie oder Perfusionsminderungen im Fluorodesoxiglucose-PET. Die Teilnehmer erhielten entweder $125 \mathrm{ml}$ einer Lösung, die eine Reihe von Nahrungsergänzungsstoffen und Vitaminen erhielt, oder entsprechendes Placebo. Die Behandlung erfolgte täglich. Als primärer Endpunkt war ein Wert auf einer umfangreichen neuropsychologischen Testbatterie definiert. Außerdem wurde die Verträglichkeit der Behandlung untersucht.

Ergebnisse: Zwischen April 2009 und Juli 2013 wurden 153 Patienten in die aktive Behandlungsgruppe und 158 in die Placebogruppe randomisiert. Die Patienten waren im Mittel 71 Jahre alt und der Wert im Mini-Mental-Test bei Studieneinschluss betrug zwischen 96,4 und 56,9. Zum Zeitpunkt des Studieneinschlusses wurden auch in der Kernspintomografie quantitativ das Volumen des Hippocampus und die Weite der Ventrikel gemessen.

Am Ende der zweijährigen Beobachtungszeit waren die Ergebnisse der neuropsychologischen Tests in beiden Gruppen vergleichbar. Insgesamt $37 \%$ der Patienten in der Kontrollgruppe und $41 \%$ in der aktiven Behandlungsgruppe entwickelten in dem zweijährigen Zeitraum eine Demenz. Die einzigen statistischen Unterschiede zugunsten der aktiven Behandlung ergaben sich für das Volumen des Hippocampus und die Weite der Ventrikel.

Schlussfolgerung: Eine zweijährige Therapie mit Nahrungsergänzungsstoffen in der Prodromalphase des Morbus Alzheimer erbringt keinen therapeutischen Nutzen.

\section{- Kommentar von Hans-Christoph Diener, Essen \\ Vorhersehbares negatives Ergebnis}

Die hier vorliegende Studie war gut geplant und durchgeführt, aber vorhersehbar negativ. Der grundsätzliche Ansatz, die Alzheimer-Erkrankung im Prodromalstadium zu behandeln, ist richtig, allerdings ist eine Zahl von 311 Patienten definitiv zu gering, wenn man berücksichtigt, dass bei vielen anderen Erkrankungen für den Therapienachweis viele Tausend Patienten benötigt werden. Außerdem ist mit hoher Wahrscheinlichkeit eine Beobachtungszeit von zwei Jahren zu gering. Da sich zumindest ein Trend zugunsten der Nahrungsergänzungsstoffe zeigte, sollte bei entsprechenden Risikopersonen jedoch auf eine gesunde Ernährung und regelmäßige sportliche Betätigung hingewirkt werden.

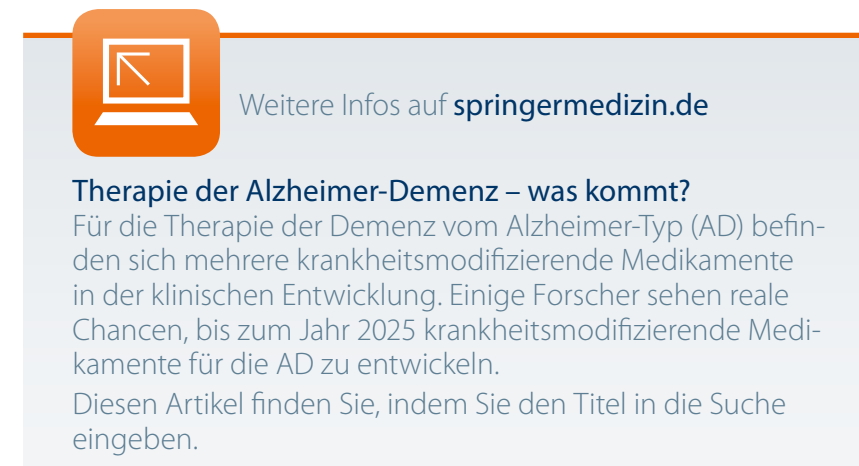

